\title{
Physical Resistance/Sticking
}

National Cancer Institute

\section{Source}

National Cancer Institute. Physical Resistance/Sticking. NCI Thesaurus. Code C133513.

Problem associated with the lack of movement in the device due parts sticking or seizing. 